\title{
Procédé enzymatique pour la détection rapide d'antibiotiques à noyau bêta lactame dans le lait
}

\author{
par \\ J. LALOUX* J. DEGELAEN ${ }^{* *}$ et P. COLART ${ }^{*}$ \\ avec la collaboration technique de P. HELLEMANS*
}

\section{Rés u mé}

Nous présentons ci-après une méthode enzymatique colorimétrique qui permet de détecter dans le lait des concentrations en antibiotiques à noyau $\beta$ lactame de l'ordre de 0,010 et $0,025 \mathrm{UI} / \mathrm{ml}$ respectivement en 20 et $10 \mathrm{~min}$.

La méthode est basée d'une part sur la propriété des antibiotiques de type $\beta$ lactame d'inhiber une enzyme D-Ala-D-Ala carboxypeptidase soluble excrétée par Actinomadura R39 et d'autre part sur la détermination de l'activité enzymatique résiduelle après interaction avec l'antibiotique.

Titre abrégé

Détection d'antibiotiques - $\beta$ lactame dans le lait.

Mots clés

Méthode enzymatique-Antibiotiques $\beta$ lactame, lait.

\section{S u m m a r y}

A rapid enzymatic colorimetric method is described which allows the detection in milk of $\beta$ lactam antibiotic concentrations of 0.010 and $0.020 \mathrm{IU} / \mathrm{ml}$ respectively in 20 and $10 \mathrm{~min}$.

\footnotetext{
* Station Laitière, chaussée de Namur, 24 B 5800 Gembloux (Belgique).

** UCB Bioproducts S.A., rue Berkendael, 68 B 1060 Bruxelles (Belgique).
} 
The method makes use of the ability of $\beta$ lactam antibiotics to inactivate a soluble D-Ala-D-Ala carboxypeptidase enzyme excreted by the R39 Actinomadura strain and rests upon the determination of residual enzyme activity after interaction with the antibiotic. Title

Detection of $\beta$ lactam antibiotics in milk.

Key words

Enzymatic - Colorimetric - Method - $\beta$ lactam antibiotics, milk.

\section{A. INTRODUCTION}

La présence d'antibiotiques dans le lait constitue un problème majeur pour l'industrie laitière.

En effet, à des taux de l'ordre de 0,010-0,050 UI/ml, les antibiotiques freinent, voire empêchent la transformation du lait en fromage et en yoghourt. Il existe, à l'heure actuelle, un grand nombre de procédés qui permettent de déceler la présence de ces antibiotiques.

Toutefois, la plupart de ces procédés, qui font appel à des techniques microbiologiques, sont très lents ( 2 à $3 \mathrm{~h}$ d'incubation) (Norme belge NBN V21-012, 1978) (Epreuve de coagulation). Récemment, a été introduit sur le marché, un test (Charm, 1980) qui permet la détermination rapide d'antibiotiques de type $\beta$ lactame.

Cette méthode fait appel à des substances radioactives et nécessite l'utilisation d'un appareillage de mesure coûteux.

FRÉRE et al., 1980 ont décrit une méthode colorimétrique de détection utilisant l'enzyme D-Ala-D-Ala carboxypeptidase soluble, excrétée par Actinomadura R39. Toutefois, cette procédure nécessite un traitement préalable du lait qui la rend complexe et de plus, le temps d'incubation est relativement long (environ $1 \mathrm{~h}$ ).

La méthode décrite ci-après est une version modifiée de celle de FrÈre et al., (1980). Elle est plus simple, plus rapide et réalisable avec du matériel de laboratoire classique. De plus, elle ne nécessite pas la pasteurisation préalable du lait, opération indispensable lorsqu'on utilise certaines méthodes microbiologiques.

\section{B. PRINCIPES THEORIQUES}

Les bases théoriques de la méthode, y compris les paramètres cinétiques qui conditionnent l'interaction entre les antibiotiques de type $\beta$ lactame et l'enzyme D-Ala-D-Ala carboxypeptidase R39 (ci-après dénommée enzyme R39) ont été décrites. (FRÈre et al., 1980). 
La plupart des antibiotiques de type $\beta$ lactame se lient rapidement à l'enzyme R39 en formant un complexe stœchiométrique covalent, inactif et sensiblement irréversible (temps de demi-vie de l'ordre de plusieurs heures). Par ailleurs, l'enzyme R39 a une activité spécifique d'hydrolyse du substrat synthétique (Ac) 2 -L-Lys-D-Ala-D-Ala avec comme résultat la libération de D-Ala.

L'activité enzymatique peut être déterminée en incubant l'enzyme en présence d'un excès de substrat et en mesurant la quantité de D-Ala libérée après un temps donné par le système D-Amino acide oxydase-flavine adénine dinucléotide - peroxydase - o - dianisidine (FRÈre et al., 1976).

La D-Ala est transformée en acide pyruvique et en eau oxygénée par le système D-Amino acide oxydase-flavine adénine dinucléotide et l'o-dianisidine réduite (incolore) est oxydée par l'eau oxygénée en présence de peroxydase en o-dianisidine oxydée qui est colorée $(\lambda \max =460 \mathrm{~nm})$.

\section{Méthode simplifiée}

Dans une première étape, 1,5 pmole d'enzyme R39 est incubée en présence de $50 \mu \mathrm{l}$ de lait pendant $5 \mathrm{~min}$ à $47^{\circ} \mathrm{C}$.

Les antibiotiques de type $\beta$ lactame éventuellement présents dans le lait se lient à l'enzyme en formant des complexes inactifs et stables dans les conditions du dosage.

Dans une seconde étape, le substrat de l'enzyme R39, à savoir I'(Ac) $)_{2}$-L-Lys-D-Ala-D-Ala, la D-Amino acide oxydase, la flavine adénine dinucléotide, la peroxydase et l'o-dianisidine sont ajoutés au mélange et l'incubation est poursuivie pendant $15 \mathrm{~min}$ à $47^{\circ} \mathrm{C}$.

Le substrat subit ou non l'hydrolyse avec libération de D-Ala.

La réaction se poursuit : l'o-dianisidine réduite est transformée en o-dianisidine oxydée.

Dans une troisième étape, de l'acide sulfurique est ajouté au mélange. Les protéines précipitent et le milieu prend une coloration rose s'il contient de l'o-dianisidine oxydée.

Dans le cas contraire, il garde une coloration jaunâtre.

- S'il n'y a pas présence d'antibiotiques de type $\beta$ lactame dans le lait, l'enzyme R39 garde toute son activité. Un maximum de D-Ala et d'o-dianisidine oxydée sera formé au cours de la seconde étape et, après addition d'acide sulfurique, le milieu d'incubation aura une coloration rose prononcée.

- Par contre, si les $50 \mu \mathrm{l}$ de lait contiennent 1,5 pmole d'antibiotiques (soit $11 \mathrm{ng}$ ou $0,017 \mathrm{UI} / \mathrm{ml}$ de lait dans le cas de la pénicilline G), l'enzyme sera complètement inhibée au cours de la $1^{\text {re }}$ étape. Il n'y aura donc pas de D-Ala, ni d'o-dianisidine oxydée 
formées au cours des étapes ultérieures et l'échantillon gardera une coloration jaunâtre après addition d'acide sulfurique.

Ce test permet donc de déterminer par simple inspection visuelle si l'échantillon contient plus ou moins de $0,017 \mathrm{UI} / \mathrm{ml}$.

Cette concentration au-delà de laquelle l'enzyme est complètement inhibée sera ci-aprés dénommée concentration limite ou seuil de détection exact. Si la concentration en antibiotiques dans le lait se situe entre 0 et $0,017 \mathrm{UI} / \mathrm{ml}$ l'échantillon présentera une coloration rose plus ou moins prononcée.

En pratique, on détermine semi-quantitativement des concentrations de l'ordre de 0,008-0,009 UI/ml. (Seuil de détection semiquantitatif).

Un des principaux avantages du test réside dans la possibilité du choix de la concentration limite et donc de la sensibilité. Ces dernières dépendent en effet du volume de lait utilisé dans le dosage. De plus, la durée du test s'en trouvera modifiée. Une diminution du volume de lait donne lieu à une diminution de la sensibilité mais aussi de la durée du test.

Des exemples pratiques sont repris dans la figure 1 et le tableau 1.

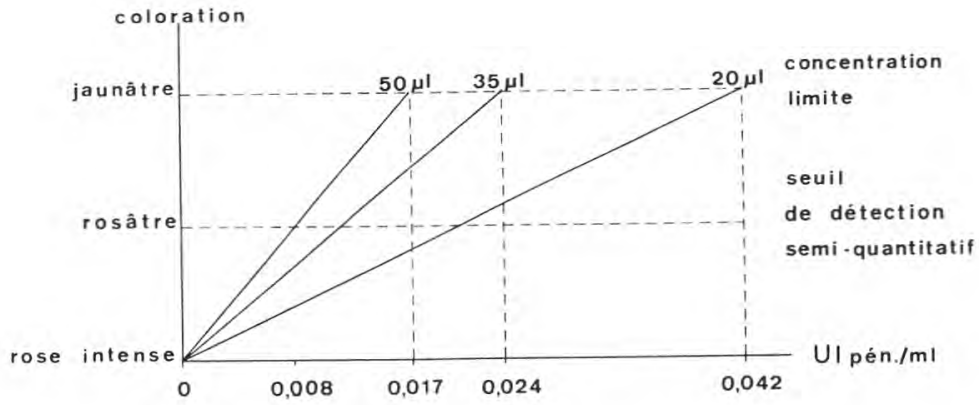

Fig. 1

Variation de l'intensité de la coloration rose obtenue en fin de dosage en fonction de la concentration en antibiotiques de type $\beta$ lactame et en fonction du volume de lait utilisé.

\section{Influence de la structure de l'antibiotique sur la sensibilité du dosage}

Nous avons dit précédemment qu'en présence de 1,5 pmole d'antibiotiques (équivalent à $0,017 \mathrm{UI} / \mathrm{ml}$ de $P e n ~ G / m l$ si on ajoute $50 \mu \mathrm{l}$ de lait au milieu d'incubation), l'enzyme R 39 est complètement inhibée si on incube le mélange pendant 5 min à $47^{\circ} \mathrm{C}$, avec comme conséquence l'obtention d'une coloration jaunâtre en fin de dosage. 
TABLEAU 1

\begin{tabular}{l|c|c|c|c}
\hline $\begin{array}{c}\text { Volume } \\
\text { de lait }\end{array}$ & \multicolumn{2}{|c|}{ Durée de la } & $\begin{array}{c}\text { Concentration } \\
\text { limite }\end{array}$ & $\begin{array}{c}\text { Seuil } \\
\text { de détection } \\
\text { semi-quantitatif }\end{array}$ \\
\hline $1^{\mathrm{e}}$ incubation & $2^{\mathrm{e}}$ incubation & $5 \mathrm{~min}$ & $0,042 \mathrm{UI} / \mathrm{ml}$ & $0,020-0,022 \mathrm{UI} / \mathrm{ml}$ \\
\hline $35 \mu \mathrm{l}$ & $5 \mathrm{~min}$ & $10 \mathrm{~min}$ & $0,024 \mathrm{UI} / \mathrm{ml}$ & $0,011-0,013 \mathrm{UI} / \mathrm{ml}$ \\
$50 \mu \mathrm{l}$ & $5 \mathrm{~min}$ & $15 \mathrm{~min}$ & $0,017 \mathrm{UI} / \mathrm{ml}$ & $0,008-0,009 \mathrm{UI} / \mathrm{ml}$ \\
\hline
\end{tabular}

Remarquons toutefois que ceci n'est vrai que pour les antibiotiques qui se lient rapidement à l'enzyme, à savoir les pénicillines V et $\mathrm{G}$, l'ampicilline, la céphaloglycine, la céphalotine, la céphalosporine et l'amoxycilline.

Avec d'autres antibiotiques tels que l'oxacilline, la cloxacilline et la méthicilline, la première étape du dosage est trop courte pour permettre leur liaison complète à l'enzyme. Il en résultera une diminution de la sensibilité du dosage pour ces antibiotiques. Ainsi par exemple, les dosages de l'oxacilline et de la cloxacilline sont respectivement trois et cinq fois moins sensibles que celui de la pénicilline G.

Il est possible cependant d'augmenter la sensibilité du dosage de ces antibiotiques en prolongeant la durée de la $1^{\text {re }}$ incubation. Si celle-ci est menée pendant $15 \mathrm{~min}$ au lieu de $5 \mathrm{~min}$ dans le dosage classique, les dosages de l'oxacilline et de la cloxacilline sont respectivement deux et trois fois moins sensibles que celui de la pénicilline G.

\section{Conduite de la détermination*}

Chaque série de tests doit contenir un échantillon témoin blanc et un échantillon témoin contrôle en plus des échantillons à tester. Pour les échantillons blanc et contrôle, on utilise un lait exempt d'antibiotiques de type $\beta$-lactame. Pour chaque échantillon, on utilise la procédure suivante :

1. A $10 \mu \mathrm{l}$ d'enzyme D-Ala-D-Ala carboxypeptidase (1,5 pmole) dans le cas des échantillons contrôle et à tester, ou à $10 \mu \mathrm{l}$ d $^{\prime} \mathrm{H}_{2} \mathrm{O}$ dans le cas de l'échantillon blanc, on ajoute $50 \mu \mathrm{l}$ de lait. On mélange et on incube pendant 5 min à $47^{\circ} \mathrm{C}$.

\footnotetext{
* La boîte Penzym ${ }^{\circledR}$ contenant les réactifs pour effectuer cent déterminations est disponible auprès de la société U.C.B. Bioproducts S.A., 68, rue Berkendael, 1060 Bruxelles (Belgique).
} 
2. On ajoute $10 \mu \mathrm{l}$ d'o-dianisidine $\left(10 \mathrm{mg} / \mathrm{ml} \mathrm{H}_{2} \mathrm{O}\right)$ et $38 \mu \mathrm{l}$ d'une solution extemporanée de substrat $\left(14 \mathrm{mg} / \mathrm{ml} \mathrm{H}_{2} \mathrm{O}\right)$, de FAD (1 mg/

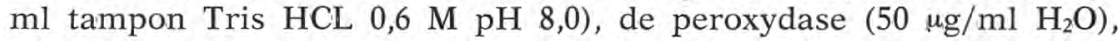
et de DAAO $\left.\left(5 \mathrm{mg} / \mathrm{ml} \text { dans } \mathrm{NH}_{4}\right)_{2} \quad \mathrm{SO}_{4} 3 \mathrm{M}\right)(10: 10: 10: 8 ; \mathrm{V} / \mathrm{V} /$ $\mathrm{V} / \mathrm{V} /)$.

On mélange et on incube pendant $15 \mathrm{~min}$ à $47^{\circ} \mathrm{C}$.

3. On ajoute $100 \mu \mathrm{l}$ de $\mathrm{H}_{2} \mathrm{SO}_{4} 50 \%$.

Les échantillons qui présentent une coloration rose franche identique à celle de l'échantillon témoin contrôle, contiennent des concentrations en antibiotiques de type $\beta$ lactame inférieures à $0,004 \mathrm{UI} / \mathrm{ml}$. Ceux qui présentent une coloration jaunâtre identique à celle de l'échantillon témoin blanc en contiennent plus de 0,017 UI/ml.

Dans les échantillons qui présentent une coloration intermédiaire la concentration en antibiotiques de type $\beta$ lactame est comprise entre 0 et $0,017 \mathrm{UI} / \mathrm{ml}$.

\section{RESULTATS EXPERIMENTAUX}

Lors des essais ci-après, nous avons toujours utilisé la dose de $50 \mu \mathrm{l}$ de lait pour les raisons suivantes :

- temps rapide d'exécution du test (un peu plus de $20 \mathrm{~min}$ ) :

- 0,008 à 0,009 UI Pénicilline $\mathrm{G} / \mathrm{ml}$ étant un seuil de détection suffisant pour des applications industrielles de la méthode.

\section{Vérification en routine du seuil de détection de la Pénicilline G dans le lait}

Dix échantillons de lait de grand mélange, auxquels on a ajouté des doses croissantes de Pénicilline G, ont été testés en double selon deux méthodes classiques (méthode d'acidification au yoghourt, méthode de diffusion en gélose suivant les normes belges) et selon la méthode enzymatique décrite ci-dessus.

Etant donné que tous les échantillons, composés de laits de régions à caractéristiques totalement différentes, ont réagi de la même manière, nous avons résumé les résultats obtenus dans le tableau 2.

Le seuil de détection mis en évidence par l'épreuve au yoghourt, effectuée avec incubation supplémentaire de $60 \mathrm{~min}$ après coagulation du témoin, est de 0,03 UI Pénicilline $\mathrm{G} / \mathrm{ml}$ de lait.

La reproductibilité des résultats a été excellente.

Les résultats obtenus à l'épreuve de diffusion en gélose montrent la croissance de la zone d'inhibition pour les trois doses examinées. 
TABLEAU 2

Détection de doses de pénicilline $\mathrm{G}$ par trois méthodes différentes

\begin{tabular}{|c|c|c|c|}
\hline $\begin{array}{l}\text { Doses de pén. } \mathrm{G} / \mathrm{ml} \\
\text { de lait }\end{array}$ & Epreuve au yoghourt & $\begin{array}{c}\text { Epreuve de diffusion } \\
\text { en gélose }\end{array}$ & $\begin{array}{c}\text { Méthode } \\
\text { enzymatique }\end{array}$ \\
\hline$+0,03$ U.I. & Début de coagulation & $\varnothing$ inhib. 22 à $23 \mathrm{~mm}$ & jaunâtre \\
\hline$+0,02$ U.I. & Coagulation & $\varnothing$ inhib. 19 à $20 \mathrm{~mm}$ & jaunâtre \\
\hline$+0,01$ U.I. & Coagulation & $\varnothing$ inhib. 15 à $16 \mathrm{~mm}$ & rosâtre \\
\hline $0 \quad$ U.I. & Coagulation & négatif & rose \\
\hline
\end{tabular}

Etant donné que l'interprétation finale du test enzymatique se fait par l'appréciation d'une coloration et que cela peut donner lieu à une interprétation différente suivant les personnes, nous avons procédé comme suit :

Après l'incubation finale, les échantillons ont été classés, tour à tour, par deux personnes :

- les roses avec l'échantillon témoin contrôle;

- les jaunâtres avec l'échantillon témoin "blanc »;

- les intermédiaires rosâtres.

Au dépouillement des résultats nous avons constaté que :

- tous les échantillons sans ajout de Pénicilline G ont été classés, avec le témoin contrôle rose ;

- tous les échantillons renfermant 0,01 UI Pénicilline $\mathrm{G} / \mathrm{ml}$ ont été classés parmi les rosâtres, avec quelques nuances de coloration .

- tous les échantillons renfermant 0,02 et 0,03 UI Pénicilline $\mathrm{G} / \mathrm{ml}$ (doses supérieures au seuil de détection exact) ont été classés sans exception dans la catégorie des jaunâtres avec le témoin «blanc " (netteté de la coloration).

Il faut également signaler que les colorations obtenues ne changent pas durant quelques heures.

La reproductibilité du test est excellente.

\section{Résultats comparatifs sur des laits à l'entrée de la laiterie}

Trente-sept échantillons de laits ont été prélevés dans des citernes à leur arrivée à la laiterie; ces échantillons ont été testés comme précédemment selon les trois méthodes.

Le tableau 3 reprend les résultats d'échantillons ayant réagi positivement à l'une ou l'autre méthode. 


\section{TABLEAU 3}

Détection des antibiotiques par 3 méthodes différentes

\begin{tabular}{|c|c|c|c|c|}
\hline $\mathrm{N}^{\circ}$ & échantillon & Epreuve au yoghourt & $\begin{array}{c}\text { Epreuve de diffusion } \\
\text { en gélose }\end{array}$ & $\begin{array}{c}\text { Méthode } \\
\text { enzymatique }\end{array}$ \\
\hline & 7 & Pas de coagulation & $\varnothing$ inhib. 17 à $18 \mathrm{~mm}$ & jaunâtre \\
\hline & 10 & Pas de coagulation & $\varnothing$ inhib. 19 à $22 \mathrm{~mm}$ & jaunâtre \\
\hline & 12 & Pas de coagulation & $\varnothing$ inhib. 16 à $17 \mathrm{~mm}$ & jaunâtre \\
\hline & 17 & Coagulation retardée & légère inhibition & rosâtre \\
\hline & 23 & Coagulation retardée & légère inhibition & rosâtre \\
\hline & 35 & Coagulation retardée & légère inhibition & rosâtre \\
\hline
\end{tabular}

Sur les 37 échantillons récoltés, 3 n'ont pas coagulé à l'épreuve au yoghourt et 3 autres ont présenté un retard à la coagulation.

Cette présence de substances freinantes a été également bien démontrée par l'épreuve de diffusion en gélose pour les 3 premiers échantillons; seulement une légère inhibition de la croissance des spores fut constatée pour les 3 derniers.

Lors du test enzymatique, les deux examinateurs classèrent les échantillons 7, 10 et 12 dans le groupe du témoin blanc, classement d'ailleurs confirmé par les deux autres méthodes, et les échantillons 17,23 et 35 dans la classe intermédiaire (rosâtre) susceptible de renfermer des traces de substances antibiotiques; ce dernier classement est confirmé par un retard de la coagulation du yoghourt et par une légère inhibition de la croissance des spores.

\section{CONCLUSION}

Le procédé enzymatique que nous avons expérimenté est un procédé rapide, pouvant être mis en œuvre sans investissement coûteux et pouvant être effectué sans pasteurisation préalable des échantillons de lait.

Ce procédé est plus sensible que les tests classiques d'acidification et est 8 à 9 fois plus rapide $(20 \mathrm{~min})$; son temps d'exécution peut encore être diminué, si l'on tolère une sensibilité moindre.

Il permet de mettre en évidence semi-quantitativement la Pénicilline $\mathrm{G}$ à partir de $0,009 \mathrm{UI} / \mathrm{ml}$, et sans équivoque à partir de dose $\geqslant 0,017 \mathrm{UI} / \mathrm{ml}$. 
Etant donné la spécificité de l'enzyme pour le noyau $\beta$ lactame, il faut s'attendre, avec cette méthode enzymatique, à un pourcentage de résultats positifs diffèrent de ceux obtenus avec les méthodes biologiques. Cependant, il faut tenir compte du fait que la Pénicilline $\mathrm{G}$ et ses dérivés, utilisés seuls ou en association avec d'autres antibiotiques, sont encore et resteront largement utilisés en thérapeutique vétérinaire.

Nous pensons que cette méthode, étant donné sa rapidité d'exécution, pourrait être utilisée avantageusement par les industriels lors du triage des laits (avant mise en fabrication de produits sensibles). Reçu pour publication en mars 1982.

\section{Bibliographie}

Charm (S. E.), (1980). - Antibiotic detection method. United States Patent, 4, $239,852$.

Frere (J. M.), Leyh-Bouille (M.), Ghuysen (J. M.), Nieto (M.) and Perkins (H. R.) (1976). - Exocellular DD Carboxypeptidase-transpeptidases from Streptomyces. Methods Enzymology, 45 B, 610-636.

FreRe (J. M.), Klein (D.) and GhuYsen (J. M.) (1980). - Enzymatic method for rapid and sensitive determination of $\beta$ lactam antibiotics. Antimicrobial Agents and Chemotherapy, 18 (4), 506-510. Service de Microbiol., Inst. de Botanique Univ. de Liège B 4000 Liège (Belgium).

Norme belge NBN V 21 - 012 (1978). - Détection de la pénicilline dans le lait par la technique des disques de papier filtre.

Ministère de l'Agriculture. Administration des Servises Economiques - Service laitier. Circulaire $\mathrm{n}^{\circ} 11$ relative à la détermination de la qualité du lait et de la crème livrés aux laiteries. Epreuve de la coagulation Annexe VI. 